\title{
Test-retest reliability of visual-evoked potential habituation \\ Test-retest reliability of visual-evoked potential habituation
}

\author{
Veronika Rauschel, Ruth Ruscheweyh, Siegbert Krafczyk \\ and Andreas Straube
}

\begin{abstract}
Objective: Habituation of visual-evoked potentials (VEPs) is typically described as deficient interictally in migraine patients, supposedly indicating altered cortical excitability. Use of this parameter for monitoring changes over time, e.g. under treatment, requires demonstration of test-retest reliability.

Methods: VEPs were recorded interictally in 4I episodic migraine patients and 40 controls. N75-PI00 amplitudes were measured over six consecutive blocks of 75 VEPs each. Amplitude regression slopes and block ratios were used to quantify VEP habituation. Test-retest reliability was assessed over 15 minutes and two to three weeks.

Results: Controls showed significantly more negative VEP habituation slopes than migraine patients $(-0.2 \mathrm{I} \pm 0.40$ vs. $0.04 \pm 0.46 \mu \mathrm{V} /$ block, $p<0.05$ ). Results were similar for block ratios, though, in the migraine group, VEP habituation significantly increased from test to two- to three-week retest $(p<0.05)$. In addition, VEP habituation test-retest correlations were mostly poor both in migraine patients and controls (intraclass correlation coefficients, 15 minutes: -0.13 to 0.30 , two to three weeks: 0.07 to 0.59 ).

Conclusions: Deficient VEP habituation in migraine was confirmed. However, the test-retest reliability of VEP habituation was rather weak. Therefore, we suggest that VEP habituation should be used for evaluation of cortical excitability under treatment only at the group level and only when a control group with sham treatment is included.
\end{abstract}

\section{Keywords}

Migraine, visual-evoked potentials, habituation, cortical excitability, test-retest reliability

Date received: 20 December 2014; revised: 31 August 2015; accepted: 18 September 2015

\section{Introduction}

Migraine is associated with considerable individual suffering and socioeconomic impact (1). It is ranked by the World Health Organization as cause number 19 of worldwide disability among all diseases (2). The underlying mechanisms are only incompletely understood. An interictal lack of habituation to sensory stimulation (e.g. visual, auditory, somatosensory and nociceptive) has been repeatedly reported (3). Also responses related to mental functions, such as contingent negative variation and P300 amplitude in oddball paradigms, show an interictal lack of habituation $(4,5)$. Therefore, the lack of habituation is seen as a major neurophysiological hallmark of migraine and has been interpreted as being the consequence of increased cortical excitability, decreased cortical inhibition or reduced cortical pre-activation. This change in cortical excitability is regarded as having a causal role in migraine pathogenesis (6). Habituation of visual-evoked potentials (VEPs) is easy to assess, cost effective, and may be of special relevance to migraine because visual symptoms are common in migraine (photophobia, visual aura) (7). For assessment of VEP habituation, pattern-reversal VEPs are usually averaged in blocks of 50-100 and amplitude slopes or first-to-last block amplitude ratios are calculated over five to six blocks (8-10). Most studies find that healthy individuals exhibit a significant habituation of VEP amplitudes while migraine patients in the interictal period do not $(8,11,12)$. There are several pharmacological and psychological interventions

University of Munich, Department of Neurology, Germany

Corresponding author:

Veronika Rauschel, Feodor-Lynen-Straße 19, D-8I377 Munich, Germany. Email: veronika.rauschel@med.uni-muenchen.de 
hypothesized to reduce migraine frequency and/or severity by regulating cortical excitability, e.g. antiepileptic drugs or psychological techniques such as progressive muscle relaxation or biofeedback (13-16). It would therefore be an obvious approach to use VEP habituation for monitoring of treatment success, for evaluation of new treatment approaches, and maybe even for individual prediction of treatment success. However, before using VEP habituation for longitudinal evaluation of treatment effects at the group level or individual level, test-retest reliability has to be demonstrated. In the present study, we confirmed group differences in VEP habituation between interictal migraine patients $(n=41)$ and healthy controls $(n=40)$ and evaluated the test-retest reliability of VEP habituation over a short period of 15 minutes ( 15 migraine patients, 15 controls) and over a longer period of two to three weeks (15 migraine patients, 24 controls). Test-retest reliability at the group level was assessed by comparing group means at test and retest. Test-retest reliability at the individual level was determined using intraclass correlation coefficients (ICCs).

\section{Methods}

\section{Individuals}

The study was conducted in accordance with the Declaration of Helsinki and approved by the local ethics committee at the University of Munich. Prior to participation, informed written consent was obtained. Patients with episodic migraine and healthy controls with normal or corrected-to-normal vision were recruited by advertisements on the hospital and university campus. Presence of episodic migraine with or without aura and absence of other headache types was diagnosed according to the current International Classification of Headache Disorders (ICHD-III beta version) (17) by an experienced physician of the outpatient headache clinic at the Department of Neurology. Healthy controls had to be free of any headaches. Participants had to be free of migraine-preventive medication for at least four weeks prior to participation. Analgesic medication and triptans were allowed, but not within 48 hours before the experiment. Migraine patients were tested interictally, meaning they were free of headache for at least 48 hours before and after the experimental session, which was assessed by a telephone or email interview 48 hours after the recording. In addition, all participants had to meet the following criteria: (1) age above 18 years, (2) sufficient knowledge of the German language, (3) no major neurological, psychiatric or medical conditions (apart from migraine in the patient group), (4) no chronic pain disorders, and (5) not pregnant or breastfeeding. All migraine patients completed the Migraine Disability Assessment Scale (MIDAS) $(18,19)$. In addition, migraine patients were interviewed regarding headache frequency (days per month in the last month), headache intensity (average during the last month) and first onset of migraine. The period of recruitment was from July 2013 until April 2014. Follow-up was conducted from April 2015 to July 2015.

Group differences and test-retest reliability were assessed. Analysis of group differences in VEP habituation was based on all migraine patients for whom at least one interictal measurement could be obtained $(n=41)$ and on all controls $(n=40)$. For test-retest analysis over 15 minutes, data from 15 interictal migraine patients and 15 controls was available and for testretest analysis over two to three weeks, data from 15 interictal migraine patients and 24 controls were available. A larger number of migraine patients had been tested for the group comparison $(n=50)$, for the testretest over 15 minutes $(n=21)$ and for the test-retest over two to three weeks $(n=31)$, but some of the migraine patients had to be excluded from analysis because they developed headache within 48 hours after the experiment. The high incidence of headache in the two- to three-week retest condition is likely due to the use of transcranial magnetic stimulation to determine magnetic suppression of visual accuracy ((20) within the same experimental sessions, but after measurement of VEPs (data reported elsewhere (21)). Occipital transcranial stimulation may have induced headaches in some of the patients (22).

\section{VEP recording}

VEP recording was performed according to international standards $(23,24)$. Participants were seated in a relaxed position in a quiet room with dimmed light. The screen was placed $135 \mathrm{~cm}$ in front of their eyes and the right eye was covered. All participants confirmed that they could see the checks clearly and got the instruction to concentrate on the screen and focus on the red fixation point in the middle of the checkerboard. During the session talking was not allowed. Standard surface electrodes (cup gold electrodes, GVB-geliMED KG, Bad Segeberg, Germany) were attached to $\mathrm{Oz}$ (active electrode) and $\mathrm{Fz}$ (reference), according to the $10 / 20$ system (25). The ground electrode was fixed on the right wrist with a ground strap. Electrode impedances were kept below $5 \mathrm{k} \Omega$. A blackand-white checkerboard reversal pattern presented on a Viewsonic E70fsB Monitor (check size 51', reversal frequency $3 \mathrm{~Hz}$, stimulated part of the visual field 14 degrees $\times 10$ degrees, Michelson's contrast 90.5\%, mean luminance $52.5 \mathrm{~cd} / \mathrm{m}^{2}$, vertical refresh rate: $60 \mathrm{~Hz}$ ) was used to evoke VEPs that were recorded using an Oxford Instruments Medical EP system (Software Version 11, Medelec Synergy, Old Woking, 
Surrey, UK). In total, 450 responses obtained by continuous stimulation were recorded between 0 and $300 \mathrm{~ms}$ after stimulation, averaged in six consecutive blocks of 75 responses and bandpass filtered from 1 to $100 \mathrm{~Hz}$. Traces were rejected automatically as artifacts if the signal amplitude exceeded $200 \mu \mathrm{V}$. The number of rejected traces was below $10 \%$ for every recording, therefore unlikely to affect the VEP habituation kinetics.

\section{VEP data analysis}

Individual N75 and P100 peaks of blocks 1 to 6 were detected automatically and confirmed visually by the investigator according to standard procedures (23). N75 to P100 peak-to-peak amplitudes were calculated and used as the measure of VEP amplitude in the present study. Linear regression over blocks 1 to 6 VEP amplitudes was used to determine the habituation slope. The ratio between the VEP amplitudes of the sixth and the first block in percentage (block ratio $=$ [block 6 amplitude/block 1 amplitude] * 100) was calculated and used as a second measure of VEP habituation. Thus, larger habituation is indicated by more negative habituation slopes and smaller block ratios. Block 1 VEP amplitudes were also analyzed to allow comparison with published data on test-retest reliability of single-block VEP amplitudes $(26,27)$.

\section{Statistics}

Statistical analysis was performed using the Statistical Package for Social Sciences (SPSS version 22, IBM Corporation, Armonk, NY, USA). Values are presented as means and standard deviations (SD) unless stated otherwise. Mean differences and correlations were considered significant if the $p$ value was $p<0.05$.
The Kolmogorov-Smirnov-test was used to test for normal distribution before the use of parametric tests. Unpaired $T$-tests were used to test for group differences in VEP block 1 amplitudes, habituation slopes and block ratios. Test-retest reliability of block 1 amplitudes, habituation slopes and block ratios was assessed (1) at the group level by comparing test and retest group mean values using paired $T$-tests and (2) at the individual level by calculating ICCs, two-way mixed, single measure, absolute agreement). Effect sizes were quantified using Cohen's $d$.

\section{Results}

\section{Group differences}

Characteristics of the study population are given in Table 1. Age and sex were not significantly different between migraine patients and controls (age: $T(79)=$ $-0.95, p=0.35$, sex: $\left.\chi^{2}(1)=0.6, p=0.43\right)$. VEP habituation profiles of migraine patients $(n=41)$ and controls $(n=40)$ are shown in Figure 1. Mean values and results of statistical tests are shown in Table 2. VEP block 1 amplitudes were not significantly different between groups. However, VEP habituation was significantly larger (i.e. more negative slopes and smaller block ratios) in controls than in migraine patients, with effect sizes (Cohen's $d$ ) of 0.39 (slope) and 0.64 (block ratio).

\section{Test-retest reliability over 15 minutes}

Test-retest reliability of block 1 amplitudes, habituation slopes and block ratios over 15 minutes was assessed in 15 migraine patients and 15 controls. Mean values and results of statistical tests are given in Table 3, and correlation plots are shown in Figure 2(a1)-(a3).

Table I. Description of the cohort. SD is given in parentheses.

\begin{tabular}{|c|c|c|c|c|c|c|}
\hline \multirow[b]{2}{*}{ Group } & \multicolumn{2}{|c|}{ Group differences } & \multicolumn{2}{|c|}{$\begin{array}{l}\text { Test-retest } \\
\text { Two to three weeks }\end{array}$} & \multicolumn{2}{|l|}{$\begin{array}{l}\text { Test-retest } \\
15 \text { minutes }\end{array}$} \\
\hline & Migraine & Control & Migraine & Control & Migraine & Control \\
\hline$n$ & 41 & 40 & 15 & 24 & 15 & 15 \\
\hline Age (years) & $30(10)$ & $28(8)$ & $35(12)$ & $26(7)$ & $31(12)$ & $31(8)$ \\
\hline Gender (male:female) & $3: 38$ & $5: 35$ & $\mathrm{I}: 14$ & $2: 22$ & $2: 13$ & $2: 13$ \\
\hline Type (with aura:without aura) & $3: 38$ & - & $0: 15$ & - & $3: 12$ & - \\
\hline Headache history (years) & $13.6(9.9)$ & - & $17.9(13.5)$ & - & $16.7(13.4)$ & - \\
\hline Headache days/month & $4.4(2.3)$ & - & 4.I (2.3) & - & $3.9(2.2)$ & - \\
\hline Headache intensity $(I-\mid 0)$ & $6.8(1.5)$ & - & $7.1(1.6)$ & - & $6.7(1.9)$ & - \\
\hline MIDAS score & $20.0(19.5)$ & - & $21.5(16.4)$ & - & $19.9(16.1)$ & - \\
\hline
\end{tabular}

MIDAS: Migraine Disability Assessment Scale. 


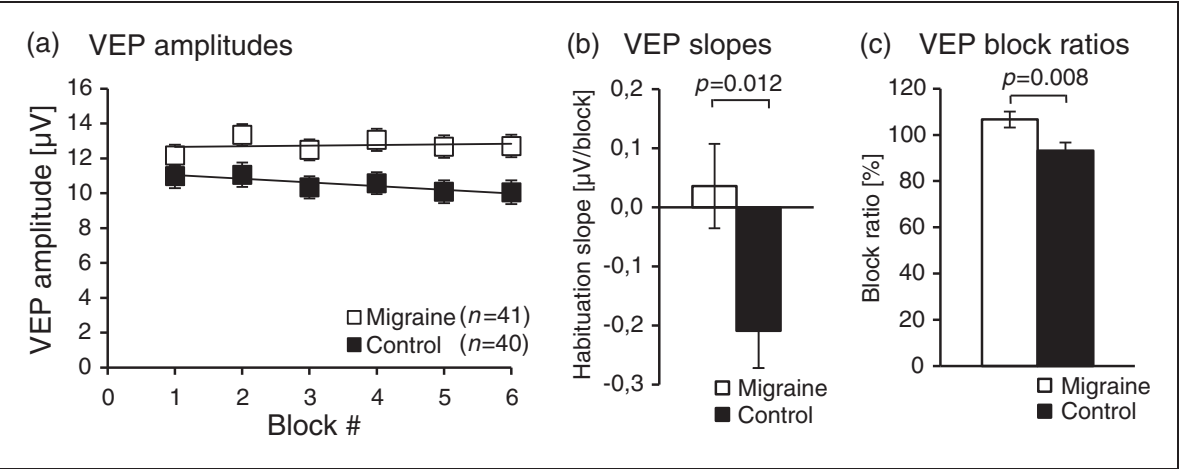

Figure I. Group differences in visual-evoked potential (VEP) habituation.

(a) VEP (N75-PI00) amplitudes (mean \pm SEM) over six blocks (75 stimuli each) are shown for healthy controls and migraine patients (measured in the interictal period). Regression lines illustrate habituation slopes in interictal migraine patients vs. controls.

(b) VEP amplitude habituation slopes (mean \pm SEM) of healthy controls and migraine patients in the interictal period are illustrated. Habituation slopes were calculated from individual block amplitudes over block I to block 6 with least squares linear regression. Habituation slopes were significantly more negative in controls than in interictal migraine patients.

(c) VEP amplitude block ratios are illustrated (mean \pm SEM). Block ratios are block 6 VEP amplitudes expressed in percentage of block I VEP amplitudes. Block ratios were significantly higher in migraine patients measured in the interictal period than in controls.

Table 2. Group comparison of visual-evoked potential (VEP) amplitudes and habituation. SD is given in parentheses. Block I amplitude indicates the first block peak to peak amplitude. Slope shows off the slope of the linear regression over block I to block 6 amplitudes. Block ratio indicates block 6 amplitude in percentage of block I amplitude.

\begin{tabular}{|c|c|c|c|c|}
\hline \multirow[b]{2}{*}{ Group } & \multicolumn{4}{|c|}{ Group differences } \\
\hline & Migraine & Control & $\begin{array}{l}T \\
P\end{array}$ & Cohen's $d$ \\
\hline$n$ & 41 & 40 & & \\
\hline Block I amplitude $(\mu \mathrm{V})$ & $12.2(3.8)$ & II $.0(4.4)$ & $\begin{array}{c}T(79)=-1.3 \\
p=0.20\end{array}$ & 0.29 \\
\hline Slope $(\mu \mathrm{V} /$ block $)$ & $0.04(0.46)$ & $-0.21(0.40)$ & $\begin{array}{c}T(79)=-2.6 \\
p=0.012\end{array}$ & 0.39 \\
\hline Block ratio (\%) & $107(22)$ & $93(22)$ & $\begin{array}{c}T(79)=-2.7 \\
p=0.008\end{array}$ & 0.64 \\
\hline
\end{tabular}

Table 3. Reliability of VEP amplitudes and habituation after 15 minutes retesting in interictal migraine patients and in controls. SD is given in parentheses. Block I amplitude indicates the first block peak to peak amplitude. Slope shows off the slope of the linear regression over block I to block 6 amplitudes. Block ratio indicates block 6 amplitude in percentage of block I amplitude.

\begin{tabular}{|c|c|c|c|c|c|c|c|c|c|c|}
\hline \multirow[b]{3}{*}{ Group } & \multicolumn{10}{|c|}{ Test-retest 15 minutes } \\
\hline & \multicolumn{5}{|l|}{ Migraine } & \multicolumn{5}{|l|}{ Control } \\
\hline & Test & Retest & $T p$ & Cohen's d & ICC & Test & Retest & $T p$ & $\begin{array}{l}\text { Cohen's } \\
d\end{array}$ & ICC \\
\hline$n$ & 15 & & & & & 15 & & & & \\
\hline $\begin{array}{l}\text { Block I } \\
\text { amplitude } \\
(\mu \mathrm{V})\end{array}$ & $12.5(3.9)$ & $12.4(4.4)$ & $\begin{array}{c}T(14)=0.2 \\
p=0.87\end{array}$ & 0.02 & $\begin{aligned} \mathrm{ICC} & =0.74 \\
P & =0.00 \mathrm{I}\end{aligned}$ & $10.1(5.0)$ & $9.0(4.2)$ & $\begin{array}{r}T(14)=1.3 \\
p=0.22\end{array}$ & 0.24 & $\begin{aligned} I C C & =0.75 \\
p & <0.00 I\end{aligned}$ \\
\hline $\begin{array}{l}\text { Slope } \\
\qquad(\mu \mathrm{V} / \text { block })\end{array}$ & $0.03(0.40)$ & $0.10(0.37)$ & $\begin{array}{c}T(I 4)=-0.6 \\
p=0.54\end{array}$ & 0.18 & $\begin{aligned} I C C & =0.30 \\
p & =0.13\end{aligned}$ & $-0.22(0.43)$ & $-0.01(0.43)$ & $\begin{array}{c}T(14)=-1.5 \\
p=0.16\end{array}$ & 0.49 & $\begin{aligned} I C C & =0.21 \\
p & =0.22\end{aligned}$ \\
\hline $\begin{array}{l}\text { Block } \\
\quad \text { ratio (\%) }\end{array}$ & $104(19)$ & II (25) & $\begin{array}{c}T(14)=-1.7 \\
p=0.12\end{array}$ & 0.59 & $\begin{aligned} \mathrm{ICC} & =-0.06 \\
p & =0.58\end{aligned}$ & 9I (24) & $106(30)$ & $\begin{array}{c}T(14)=-1.5 \\
p=0.16\end{array}$ & 0.55 & $\begin{aligned} I C C & =-0.13 \\
p & =0.69\end{aligned}$ \\
\hline
\end{tabular}

VEP: visual-evoked potential; ICC: intraclass correlation coefficient. 


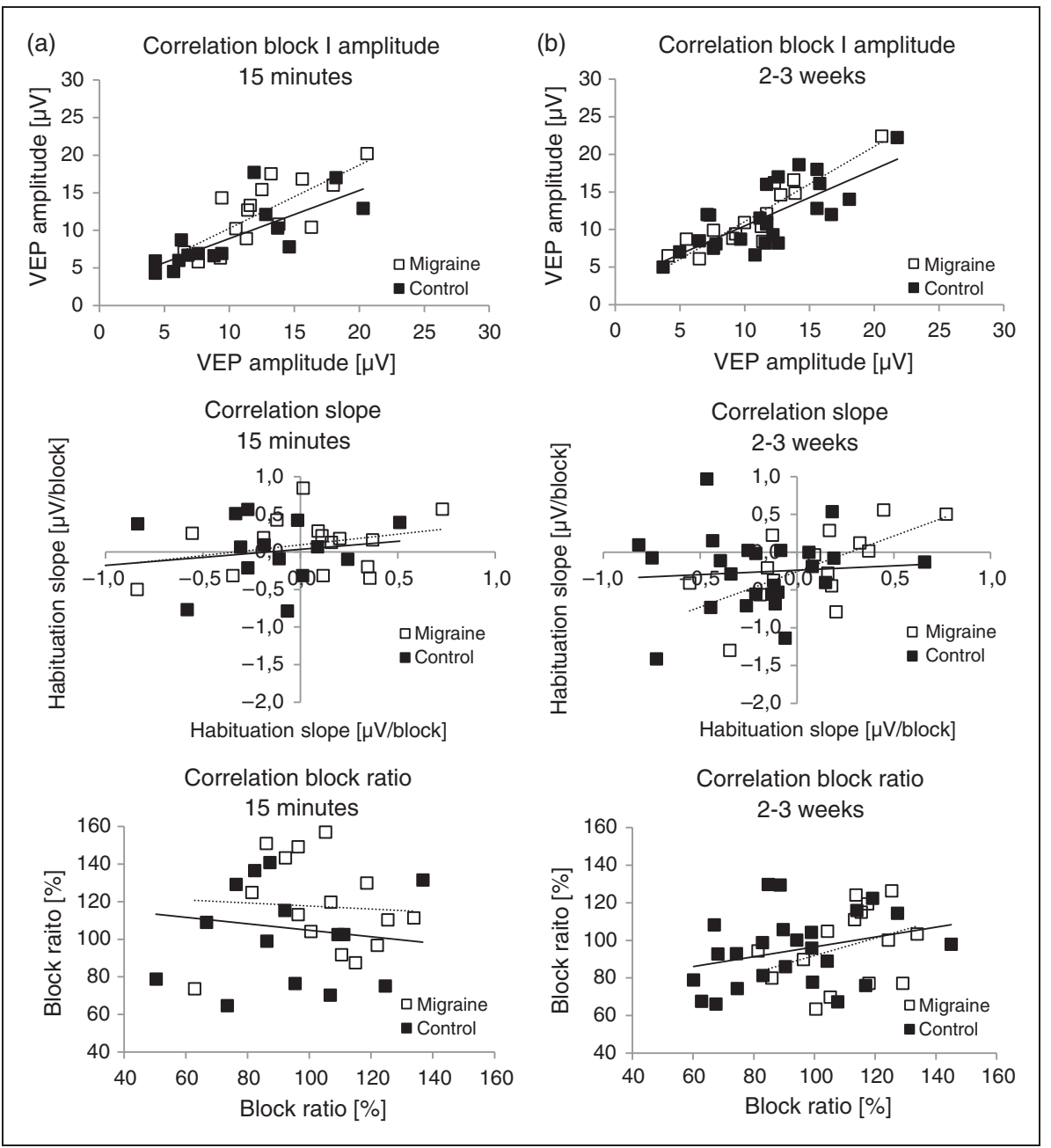

Figure 2. Test-retest reliability of visual-evoked potential (VEP) measures.

(a I)-(a3) Test-retest reliability over 15 minutes (I5 migraine patients, I5 controls). Correlations between VEP measures at test and retest are illustrated. Linear regression lines are shown.

(bl)-(b3) Test-retest reliability over two to three weeks (I 5 migraine patients and 24 controls). Correlations between VEP measures at test and retest are illustrated. Linear regression lines are shown.

VEP measures were as follows: Block I amplitudes are first-block VEP (N75-PI00) amplitudes. Habituation slopes were calculated from individual VEP block amplitudes over block I to block 6 with least squares linear regression. Block ratios were calculated as block 6 VEP amplitudes expressed in percentage of block I VEP amplitudes.

In controls, mean values at test and retest were similar for all parameters $(p>0.1)$. Correlations between test and retest values were high for block 1 amplitudes $(\mathrm{ICC}=0.75)$ but low for habituation slopes and block ratios $(\mathrm{ICC}=0.21$ and -0.13 , respectively). Similarly, there were also no significant differences between test and retest values for any of the tested parameters in migraine patients $(p>0.1)$. Test-retest correlations in migraine patients were high for block 1 amplitudes $(\mathrm{ICC}=0.74)$, but low for habituation slopes $(\mathrm{ICC}=$ $0.30)$ and for block ratios $(\mathrm{ICC}=-0.06)$. Although none of the differences reached statistical significance, it must be noted that both in the migraine and control groups, habituation slopes were less negative at the 15-minute retest, and block ratios were increased. Effect sizes reached values between 0.18 and 0.59 , which is partially within the range of effect sizes found for the difference between migraine patients and controls $(d=0.39$ and 0.64 , see Table 2$)$.

\section{Test-retest reliability over two to three weeks}

Test-retest reliability of block 1 amplitudes, habituation slopes and block ratios over two to three weeks was assessed in 15 migraine patients and 24 controls. Mean values and results of statistical tests are given in Table 4, and correlation plots are shown in Figure 2(b1)-(b3). In controls, mean values at test 
Table 4. Reliability of VEP amplitudes and habituation after two to three weeks retesting in interictal migraine patients and in controls. SD is given in parentheses. Block I amplitude indicates the first block peak to peak amplitude. Slope shows off the slope of the linear regression over block I to block 6 amplitudes. Block ratio indicates block 6 amplitude in percentage of block I amplitude.

\begin{tabular}{|c|c|c|c|c|c|c|c|c|c|c|}
\hline \multirow{4}{*}{ Group } & \multicolumn{10}{|c|}{ Test-retest two to three weeks } \\
\hline & \multicolumn{5}{|l|}{ Migraine } & \multicolumn{5}{|l|}{ Control } \\
\hline & & & $T$ & Cohen's & & & & $T$ & Cohen's & \\
\hline & Test & Retest & $p$ & $d$ & ICC & Test & Retest & $p$ & $d$ & ICC \\
\hline$n$ & 15 & & & & & 24 & & & & \\
\hline $\begin{array}{l}\text { Block I } \\
\quad \text { amplitude }(\mu \mathrm{V})\end{array}$ & $10.7(4.1)$ & II.7 (4.4) & $\begin{array}{c}T(I 4)=-2.3 \\
p=0.04\end{array}$ & 0.24 & $\begin{aligned} I C C & =0.91 \\
p & <0.00 I\end{aligned}$ & II.6 (4.4) & II.7 (4.4) & $\begin{array}{c}T(23)=-0.2 \\
p=0.88\end{array}$ & 0.02 & $\begin{aligned} \mathrm{ICC} & =0.75 \\
p & <0.00 \mathrm{I}\end{aligned}$ \\
\hline $\begin{array}{l}\text { Slope } \\
\qquad(\mu \mathrm{V} / \text { block })\end{array}$ & $0.08(0.34)$ & $-0.18(0.50)$ & $\begin{array}{c}T(I 4)=2.6 \\
p=0.02\end{array}$ & 0.61 & $\begin{aligned} I C C & =0.59 \\
p & =0.008\end{aligned}$ & $-0.19(0.33)$ & $-0.26(0.50)$ & $\begin{array}{c}T(23)=0.6 \\
p=0.56\end{array}$ & 0.17 & $\begin{aligned} \mathrm{ICC} & =0.07 \\
p & =0.37\end{aligned}$ \\
\hline Block ratio (\%) & III (I5) & $97(20)$ & $\begin{array}{c}T(14)=2.6 \\
p=0.02\end{array}$ & 0.79 & $\begin{aligned} I C C & =0.34 \\
p & =0.10\end{aligned}$ & $93(22)$ & $95(19)$ & $\begin{array}{c}T(23)=-0.4 \\
p=0.69\end{array}$ & 0.10 & $\begin{aligned} I C C & =0.30 \\
p & =0.07\end{aligned}$ \\
\hline
\end{tabular}

VEP: visual-evoked potential; ICC: intraclass correlation coefficient.

and retest were similar for all parameters $(p>0.5)$. Correlations between test and retest values were high for block 1 amplitudes (ICC $=0.75$ ) but low for habituation slopes and block ratios $(\mathrm{ICC}=0.07$ and 0.30 , respectively). For migraine patients, block 1 amplitudes were significantly higher at retest and habituation slopes and block ratios were significantly lower at retest (all $p<0.05$ ). Test-retest correlations were high for block 1 amplitudes $(\mathrm{ICC}=0.91)$, moderate for habituation slopes $(\mathrm{ICC}=0.59)$ and low for block ratios $(\mathrm{ICC}=0.34)$.

Habituation slopes between test and retest after 15 minutes and after two to three weeks were slightly different. In controls, habituation slopes tended to be more negative at the 15-minute test than at the retest $($ slope $($ test $)=-0.22 \pm 0.43 ; \quad$ slope $($ retest $)=-0.01 \pm 0.43)$ $(p=0.16)$. Migraine patients showed no difference at the retest after 15 minutes compared to the first experimental session $\quad($ slope $($ test $)=0.03 \pm 0.40 ; \quad$ slope $($ retest $)=0.10$ $\pm 0.37)(p=0.54)$.

Habituation slopes for test and retest after two to three weeks showed different results. Both groups showed more habituation at the retest session, but this was not significantly different for controls. In contrast, migraine patients showed a significantly different habituation between test and retest (controls: slope(test) $=$ $-0.19 \pm 0.33, \quad$ slope $($ retest $)=-0.26 \pm 0.50 \quad(p=0.56)$; migraine patients: slope $($ test $)=0.08 \pm 0.34$, slope $($ retest $)=$ $-0.18 \pm 0.50(p=0.020))$.

\section{Discussion}

Main results of the present study are: (1) deficient interictal VEP habituation was confirmed in episodic migraine patients, (2) VEP habituation group mean values showed significantly increased habituation in the migraine group at the two- to three-week retest, and (3) correlations between VEP habituation measures at test and retest were disappointing, both for the 15 minutes and the two- to three-week interval.

To the best of our knowledge, test-retest reliability of VEP habituation has not been assessed before. Testretest reliability of VEP P100 latencies has been assessed before and yielded test-retest correlation coefficients around 0.7 over an unspecified interval (28) and of $0.59-0.93$ over eight months (27). Test-retest reliability of VEP N75-P100 amplitudes (a single block of 100 trials) has been shown to be good over eight months $(r=0.72)(27)$. This is consistent with the results of the present study, in which test-retest ICCs of block 1 VEP amplitudes were above 0.74 for both test-retest intervals and both groups. In contrast, test-retest correlations of VEP habituation measures were mostly poor (ICC 0.07 to 0.59 for two to three weeks) in the present study, suggesting that VEP habituation may not be the best way to monitor individual changes of cortical excitability over time. Previous studies have shown that cortical excitability and habituation are dependent on the migraine cycle, with a maximum habituation deficit immediately before the attack and normalization of the habituation during the attack $(4,29)$. These studies showed that every patient may be driven by his or her own "migraine-rhythm." Therefore, we hypothesized that the poor test-retest correlations found over the two- to three-week interval might be due to unpredictable changes of cortical excitability during the individually varying migraine cycle (although all measurements were conducted in the interictal period as documented by telephone interview two days after the recordings). To exclude this possibility, we conducted an additional set of experiments investigating test-retest reliability over 15 minutes. However, 
correlations between VEP habituation at baseline and 15 minutes later were also poor (ICC 0.06 to 0.30 ), suggesting a large inherent variability of VEP habituation as quantified by habituation slopes and block ratios instead of physiological changes over time as the cause of poor test-retest correlations.

Other measures of cortical excitability also differentiate between migraine patients and controls and may exhibit better test-retest reliability than VEP habituation. Contingent negative variation (CNV) amplitudes are increased in migraine patients compared to controls $(4,30)$. Test-retest reliability of CNV over 10 days has been examined by Kropp et al. (31) in 27 healthy volunteers. Test-retest correlation coefficients were good, especially for the early $\mathrm{CNV}$ component (iCNV, Pearson's $r=0.86$ ). The authors concluded that the standardized $\mathrm{CNV}$ recording procedure is reproducible and stable in healthy individuals. Therefore, the early CNV component may be more suitable to follow individual changes over time than VEP habituation, but this has not been shown until now in migraine patients.

In addition to mostly poor test-retest correlations of VEP habituation, our results also indicate that group mean values of VEP habituation measures may not necessarily be stable over time. Importantly, there was a significant increase in habituation in the migraine group over the two- to three-week interval. For the 15-minute interval, a possible systematic shift toward smaller habituation at retest compared to test was observed both in the migraine and the control groups, which has to be interpreted with much caution because it did not reach significance. The reasons for these shifts over time are not clear. It has been described that habituation may increase after repeated stimulation (32). This might in part explain the increase in habituation in the migraine group after two to three weeks, but is at variance with the possible shift toward smaller habituation over 15 minutes. However, in most experimental settings, net results of habituation are caused by the sum of concurrent habituation and sensitization processes that occur with different kinetics and therefore may give different results depending on the tested time frame ("dual-process theory" (32)). In migraine, it is strongly debated if the lack of net habituation seen, e.g. in VEP recordings, is the result of increased cortical excitability (leading to decreased habituation) or the result of decreased cortical pre-activation levels (leading to decreased VEP amplitudes at stimulation onset, which implies that according to the "ceiling model" of cortical excitation, the threshold for initiation of habitation is reached later in migraine patients than controls) $(6,33)$. Therefore, habituation in migraine might also involve a combination of both mechanisms, possibly with different kinetics.
Several studies have monitored VEP amplitudes or habituation in parallel with clinical outcome during preventive migraine treatment (34-36). Diener et al. (34) recorded a single block (64 trials) of VEPs before and after four months of preventive treatment with beta blockers in 41 migraine patients. Migraine patients had significantly higher VEP amplitudes than healthy volunteers at baseline. The use of beta blockers led to a significant decrease in VEP amplitude in migraine patients, but was not related to the clinical treatment effect. After three months of washout, VEP amplitudes partially returned to the initial values. VEP habituation was not tested in this study. Ozkul and Bozlar (35) monitored the effect of fluoxetine on interictal VEP habituation (five blocks of 50 trials) in 79 migraine patients. At baseline, VEP block ratios were significantly higher in migraine patients compared to controls. After four weeks of fluoxetine, VEP block ratios were similar to the baseline values of the control group. A correlation with the clinical effect was not reported. One study also examined treatment effects over a 15-minute interval. Viganò et al. (36) recorded VEP habituation slopes (over six blocks of 100 trials) before and after 15 minutes of anodal transcranial direct current stimulation (tDCS) over the visual cortex, and again three hours later in 11 controls and 13 interictal migraine patients. VEP habituation slopes were significantly more negative after tDCS both in migraine patients and controls, and back to baseline values after three hours. Our data suggest that there may be a systematic shift in VEP habituation at the retest interval of 15 minutes, which may compromise the interpretation of treatment effects at this time window. However, in the cited study (36) the treatment effect was in the direction of increased habituation, while the systematic shift detected in the present study was in the direction of reduced habituation, suggesting that the effect of tDCS on VEP habituation was real. Nonetheless, our results, showing that VEP habituation is not necessarily stable over time, underline that treatment studies should always include a group with sham treatment or without treatment to determine the natural course of VEP habituation over the investigated time interval.

To the best of our knowledge, the present study is the first to investigate test-retest reliability of VEP habituation. An important limitation was the relatively small number of participants in the test-retest part of the study. However, the number was sufficient to show a significant increase in VEP habituation in migraine patients over the two- to three-week interval. Another limitation is the fact that we pooled together migraine patients with and without aura. However, there were only three migraine patients with aura, suggesting that their inclusion cannot affect fundamentally the results. 
A strength of the study is that participants of both groups were recruited from the general population. Including patients from a tertiary care facility might significantly bias the results because these patients are usually severely affected. A further strength of the study is the care that was taken to include individuals only during the interictal period (48 hours before and after the measurement). A further limitation is the relatively low mean age in the present study, limiting the generalizability of the results to older individuals. However, migraine is a disorder that is most prevalent in younger adults (37).

\section{Conclusion}

VEP habituation is not generally suitable to assess longitudinal changes of cortical excitability at an individual level. It may be acceptable for monitoring treatment effects at the group level, provided the group size is large enough, and a control group without treatment or with sham treatment is included since the VEP habituation may also change in these patients over time.

\section{Article highlights}

- A significantly larger visual-evoked potential (VEP) habituation was found in controls compared to interictal migraine patients.

- VEP habituation in migraine patients showed a significant shift toward larger habituation at the two- to three-week retest interval, indicating that a sham treatment group should be included in studies using VEP habitation as a measure of treatment outcome.

- Test-retest reliability of VEP habituation was insufficient to follow individual changes of cortical excitability over time.

\section{Acknowledgments}

We would like to thank all participants of this study (as well as Mrs. K. Ogston for editing the paper). The study was approved by the local ethics committee at the University of Munich (Project number: 133-13). Participants gave informed consent before taking part.

\section{Declaration of conflicting interests}

The authors declared no potential conflicts of interest with respect to the research, authorship, and/or publication of this article.

\section{Funding}

This work was supported by a grant to VR from the DFG (GRK 1091).

\section{References}

1. Rasmussen BK. Epidemiology and socio-economic impact of headache. Cephalalgia 1999; 19(Suppl 25): 20-23.

2. Levav I and Rutz W. The WHO World Health Report 2001 new understanding-new hope. Isr J Psychiatry Relat Sci 2002; 39: 50-56.

3. Harriott AM and Schwedt TJ. Migraine is associated with altered processing of sensory stimuli. Curr Pain Headache Rep 2014; 18: 458.

4. Kropp P and Gerber WD. Prediction of migraine attacks using a slow cortical potential, the contingent negative variation. Neurosci Lett 1998; 257: 73-76.

5. Wang W and Schoenen J. Interictal potentiation of passive "oddball" auditory event-related potentials in migraine. Cephalalgia 1998; 18: 261-265. discussion 241.
6. Coppola G, Pierelli F and Schoenen J. Habituation and migraine. Neurobiol Learn Mem 2009; 92: 249-259.

7. Cucchiara B, Datta R, Aguirre GK, et al. Measurement of visual sensitivity in migraine: Validation of two scales and correlation with visual cortex activation. Cephalalgia 2015; 35: 585-592.

8. Afra J, Cecchini AP, De Pasqua V, et al. Visual evoked potentials during long periods of pattern-reversal stimulation in migraine. Brain 1998; 121: 233-241.

9. Afra J, Ambrosini A, Genicot R, et al. Influence of colors on habituation of visual evoked potentials in patients with migraine with aura and in healthy volunteers. Headache 2000; 40: 36-40.

10. Coppola G, Cremers J, Gerard P, et al. Effects of light deprivation on visual evoked potentials in migraine without aura. BMC Neurol 2011; 11: 91.

11. Coppola G, Parisi V, Di Lorenzo C, et al. Lateral inhibition in visual cortex of migraine patients between attacks. J Headache Pain 2013; 14: 20.

12. Bednar M, Kubova Z and Kremlacek J. Lack of visual evoked potentials amplitude decrement during prolonged reversal and motion stimulation in migraineurs. Clin Neurophysiol 2014; 125: 1223-1230.

13. Ferraro D, Vollono C, Miliucci R, et al. Habituation to pain in "medication overuse headache": $\mathrm{A} \mathrm{CO}_{2}$ laserevoked potential study. Headache 2012; 52: 792-807.

14. Di Clemente L, Puledda F, Biasiotta A, et al. Topiramate modulates habituation in migraine: Evidences from nociceptive responses elicited by laser evoked potentials. J Headache Pain 2013; 14: 25.

15. Andrasik F. Biofeedback in headache: An overview of approaches and evidence. Cleve Clin J Med 2010; 77(Suppl 3): S72-S76. 
16. Nestoriuc Y, Martin A, Rief W, et al. Biofeedback treatment for headache disorders: A comprehensive efficacy review. Appl Psychophysiol Biofeedback 2008; 33: 125-140.

17. Headache Classification Committee of the International Headache Society. The International Classification of Headache Disorders, 3rd edition (beta version). Cephalalgia 2013; 33: 629-808.

18. Stewart WF, Lipton RB, Kolodner K, et al. Reliability of the migraine disability assessment score in a populationbased sample of headache sufferers. Cephalalgia 1999; 19: 107-114. discussion 74.

19. Stewart WF, Lipton RB, Whyte J, et al. An international study to assess reliability of the Migraine Disability Assessment (MIDAS) score. Neurology 1999; 53: 988-994.

20. Mulleners WM, Chronicle EP, Palmer JE, et al. Suppression of perception in migraine: Evidence for reduced inhibition in the visual cortex. Neurology 2001; 56: 178-183.

21. Rauschel V, Ruscheweyh R, Eggert T, et al. Magnetic suppression of perceptual accuracy is not reduced in episodic migraine without aura. J Headache Pain 2014; 15: 83.

22. Maizey L, Allen CP, Dervinis M, et al. Comparative incidence rates of mild adverse effects to transcranial magnetic stimulation. Clin Neurophysiol 2013; 124: 536-544.

23. Odom JV, Bach M, Barber C, et al. Visual evoked potentials standard (2004). Doc Ophthalmol 2004; 108: 115-123.

24. Marmor MF, Hood DC, Keating D, et al. Guidelines for basic multifocal electroretinography (mfERG). Doc Ophthalmol 2003; 106: 105-115.

25. American Electroencephalographic Society. Guideline thirteen: Guidelines for standard electrode position nomenclature. American Electroencephalographic Society. J Clin Neurophysiol 1994; 11: 111-113.

26. Schellberg D, Gasser T and Köhler W. The intra-individual reproducibility of flash-evoked potentials in a sample of children. Int J Psychophysiol 1987; 5: 135-143.
27. Sarnthein J, Andersson M, Zimmermann MB, et al. High test-retest reliability of checkerboard reversal visual evoked potentials (VEP) over 8 months. Clin Neurophysiol 2009; 120: 1835-1840.

28. Hacke W. Visual evoked potentials from the viewpoint of test theory [article in German]. EEG EMG Z Elektroenzephalogr Elektromyogr Verwandte Geb 1985; 16: 158-161.

29. Judit A, Sandor PS and Schoenen J. Habituation of visual and intensity dependence of auditory evoked cortical potentials tends to normalize just before and during the migraine attack. Cephalalgia 2000; 20: 714-719.

30. Siniatchkin M, Averkina N, Andrasik F, et al. Neurophysiological reactivity before a migraine attack. Neurosci Lett 2006; 400: 121-124.

31. Kropp P, Kiewitt A, Göbel H, et al. Reliability and stability of contingent negative variation. Appl Psychophysiol Biofeedback 2000; 25: 33-41.

32. Rankin $\mathrm{CH}$, Abrams $\mathrm{T}$, Barry RJ, et al. Habituation revisited: An updated and revised description of the behavioral characteristics of habituation. Neurobiol Learn Mem 2009; 92: 135-138.

33. Bohotin V, Fumal A, Vandenheede M, et al. Effects of repetitive transcranial magnetic stimulation on visual evoked potentials in migraine. Brain 2002; 125: 912-922.

34. Diener HC, Scholz E, Dichgans J, et al. Central effects of drugs used in migraine prophylaxis evaluated by visual evoked potentials. Ann Neurol 1989; 25: 125-130.

35. Ozkul Y and Bozlar S. Effects of fluoxetine on habituation of pattern reversal visually evoked potentials in migraine prophylaxis. Headache 2002; 42: 582-587.

36. Viganò A, D’Elia TS, Sava SL, et al. Transcranial direct current stimulation (tDCS) of the visual cortex: A proofof-concept study based on interictal electrophysiological abnormalities in migraine. J Headache Pain 2013; 14: 23.

37. Haan J, Hollander $\mathbf{J}$ and Ferrari MD. Migraine in the elderly: A review. Cephalalgia 2007; 27: 97-106. 